\title{
Modular Phased Array Ultrasound Doppler Platform for Measurements in Liquid Metals
}

\author{
Hannes Beyer, Richard Nauber, Norman Thieme, Lars Büttner, Jürgen Czarske \\ Laboratory of Measurement and Sensor System Techniques, Faculty of Electrical and Computer \\ Engineering, School of Engineering, TU Dresden 01062 Dresden, Germany \\ http://www.lasermetrology.de \\ E-mail: hannes.beyer@mailbox.tu-dresden.de
}

\begin{abstract}
The quality of silicon ingots for photovoltaics can be improved by applying time-varying magnetic fields during the solidification process. Their influence on the material and heat transfer in the molten silicon is investigated by means of numerical simulation and laboratory-scale model-experiments with lowmelting metals. Ultrasound Doppler velocimetry is suitable for flow investigations of opaque liquids. While conventional systems using transducers with fixed sound field provide only limited resolution, our approach is to use a phased array (PA) with the ability to focus and steer its acoustic field. We present a phased array ultrasound Doppler velocimeter (PAUDV) for flow mapping and novel measurement methods like two-point correlation of turbulent flows. The PAUDV is a modular platform with 256 independent controllable channels. Each channel is capable of transmitting pulse bursts with a peak-to-peak amplitude of $200 \mathrm{~V}$, a frequency typically ranging between 1 and $10 \mathrm{MHz}$ and a burst length of one to sixteen periods. The bursts across all channels can be aligned and delayed with a resolution of $1.6 \mathrm{~ns}$ to each other with a maximum repition frequency of approx. $60 \mathrm{kHz}$. The PAUDV is a modular system consisting of two backplanes, one master card (control module) and up to eight transceiver cards with 32 channels each. A measurement example of the sound field from a designed phased array is given, which shows that the achievable lateral resolution is better than $1.5 \mathrm{~mm}$ for that system configuration.
\end{abstract}

Key words: Phased Array, Multiplex Technique, FPGA, System Characterization, Flow Mapping, Magnetohydrodynamics

\section{Introduction}

Flow control is the key to improve the quality, energy efficiency and other important factors of industrial processes that involve metal melts. A promising approach is to affect electrically conductive fluids with magnetic fields, using the insights from the research field of magnetohydrodynamics (MHD). In order to improve processes such as semiconductor crystal growth and continuous steel casting, a profound understanding of the relation between magnetic fields, induced Lorentz forces and their effect on the liquid flow is crucial. Numerical models are typically used to investigate that complex interaction, requiring model experiments for their validation and refinement. To model the hot melts at room temperature, opaque liquid metals like galliumindium-tin (GalnSn) are commonly used, which require appropriate flow instrumentation systems. For multi plane, multi component flow mapping of these opaque liquids, instrumentation systems like the ultrasound array
Doppler velocimeter (UADV) are available ${ }^{[1,2]}$. However, other experiments demand high, submillimeter resolution scans of the flow as well as measuring two velocity components through one acoustical access. Further, advanced measurement methods like two-point correlation functions of turbulent liquid metal flows are currently not applicable. In order to address these experiments, it is necessary to electrically shape and tilt the sound field of the ultrasound (US) sensor, known as phased array beamforming (BF) ${ }^{[3]}$. This technique uses electrical pulses with fine adjustable delays for each ultrasound element of an array with up to hundreds of elements. The elements have subwavelength dimensions, emitting a shaped sound field through overlaying all single emissions by an interference principle.

We present a phased ultrasound array Doppler velocimeter system for high precision measurements in liquid metals requiring electrical steering and focusing of the acoustical field. The modular design enables an operation 
with up to 256 elements, providing an individual programmable delay for beamforming operation on transmit side.

\section{Architecture of the Measurement System}

The PAUDV is separated into functional blocks, which are shown in figure 1. The system consists of eight 32-channel ultrasound transceiver modules containing a full analog front end for receiving and a binary high voltage (HV) driver front end for transmitting ultrasound signals. The transmission path is driven by the transmission (TX) beamformer, which synthesizes the delayed pulse patterns in a two bit binary fashion for each channel. These two control bits represent a digital waveform with three amplitude states $+1,0$ and -1 . They correspond to the pulse voltages $U_{H V_{+}}$, zero and $U_{H V}$. and are generated in the HV pulse driver circuit. The HV signals are routed through a transmit/receive switch to the HV backplane, collecting and distributing all 256 channels on one printed circuit board to a custom defined number $n$ of ultrasound arrays. The signal distribution can be programmed through the main controller module using a customized HV Backplane. From the HV backplane, the signals are routed back through their corresponding $T / R$ switches to the pre-amplifier stage. It performs the essential low-noise signal conditioning for the external analog-digital (AD) conversion process.

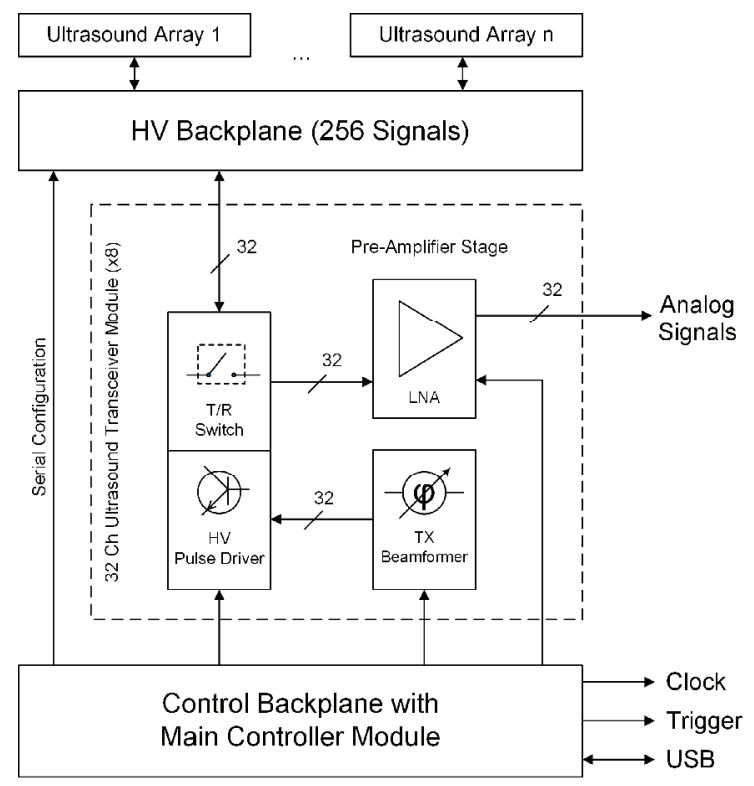

Figure 1: Modular Architecture of the PAUDV

An external backend consisting of a FPGA (NI PXle 7965R) and an AD Converter Module (NI 5752) digitizes and processes the signals. The individual delays of the excitation signals for all elements are programmed by the main controller module. It serves as a transparent interface between an external host personal computer (PC) and the TX beamformers with further abilities to configure the HV backplane as well as the amplifiers and HV pulse drivers on the transceiver modules. The host PC communicates with the main controller via a universal serial bus (USB) connection. The host sends instructions to the PAUDV, thus controlling the behavior of the measurement system. In order to obtain high scanning frequencies, the main controller provides a beamformer data cache and a generic instruction cache to efficiently progress through the measurement program for time-critical parts of the measurement without communication latency. New beamformer data, i.e. delay rules for a focus point can be updated through specific instructions and its parameters. We implemented a streaming functionality for the configuration data in order to measure over a long period of time. This is achieved by internally buffer the instruction data in a ring structure, enabling a block-wise download and execution of the measurement program.

\section{Functional Specification}

The PAUDV is able delay each channel in fine steps of $1.6 \mathrm{~ns}$ with a common pulse frequency ranging between 1.0 and $10 \mathrm{MHz}$ and a pulse length of up to 32 periods. The amplitude can be as high as $200 \mathrm{~V}_{\mathrm{p}-\mathrm{p}}$ with a symmetrical power supply design of $+/-100 \mathrm{~V}$. At the receiving path, the signals are amplified by a programmable gain ranging from $17 \mathrm{~dB}$ to $67 \mathrm{~dB}$. The configuration rate of the TX beamformers, i.e. changing the individual delays of all elements, can be performed with a rate of up to $60 \mathrm{kHz}$.

\section{Ultrasound Sensors}

A customizable HV backplane allows interfacing to a wide range of sensors from a set of single transducers to planar phased arrays. The current implementation of the backplane (HVHT1) uses a 128 channel Hypertronics connector that is common for commercially available PAs. Furthermore, a multiplexing backplane (HVMUX1) was designed to increase the number of channels by using 1:4 multiplex and programmable channel damping for a total of 256 channels.

Beside the phased array operation, the PAUDV is also capable of performing conventional pulsed wave Doppler (PWD) velocimetry (i.e. performed by the UADV ${ }^{[4]}$ ). For linear phased arrays and a given delay rule, the shape of the sound field is a function of the delay resolution, the pulse frequency provided by the PAUDV, the velocity of sound in the instrumentation fluid 
and the geometric dimensions of the individual array. We simulated the resulting sound field for such a given set of parameters. Figure 2 shows a simulation of a linear phased array with a US frequency $f=3 \mathrm{MHz}$ and an element pitch of $d_{p}=0.5 \mathrm{~mm}^{[5]}$.

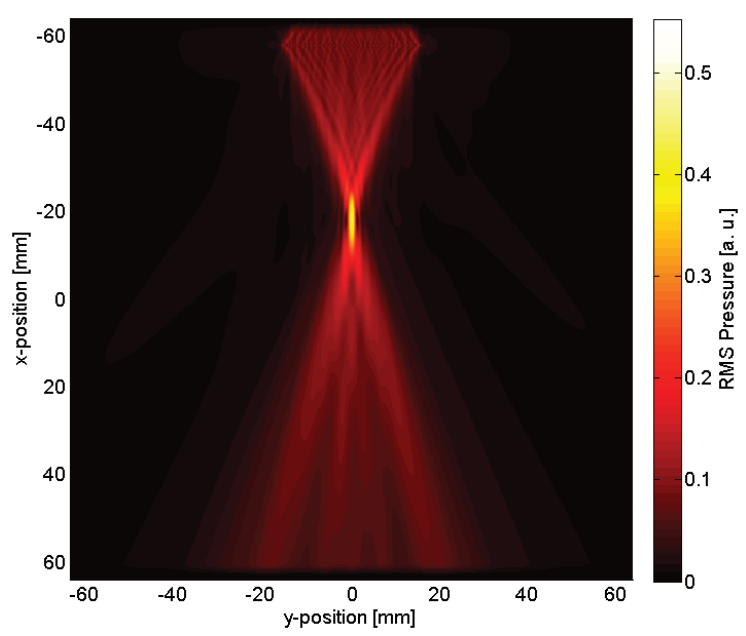

Figure 2: Simulated sound field of a linear phased array with $n=64$ elements and $F=40 \mathrm{~mm}$

\section{Sensor for Measuring in Liquid Metal}

For investigation in GalnSn with an acoustic velocity of $c_{G a l n S n}=2670 \mathrm{~m} / \mathrm{s}$, a linear phased array with $n=128$ elements, operating frequency of $f=3 \mathrm{MHz}$ and an element pitch of $d_{p}=0.5 \mathrm{~mm}$ is used. It has been designed to achieve a lateral resolution of approximately $1 \mathrm{~mm}$ at a distance $F=40 \mathrm{~mm}$. It is acoustically matched to the sound velocity of PMMA, a commonly used material for containers with GalnSn. For the characterization, the sensor was mounted onto a container of water to measure the sound field of the phased array. Since water has a different sound velocity than GalnSn $\left(c_{\text {Water }}=1484 \mathrm{~m} / \mathrm{s}\right)$, the calculation of the delay rules have been adjusted for the characterization.

\section{Characterization of the PAUDV}

In order to prove the phased array functionality of the PAUDV with the given sensor, we aligned a thin wire with a diameter of $d=120 \mu \mathrm{m}$ in parallel to the z-axis, defining the direction of ultrasound emission as $y$-axis and the lateral direction as the $x$-axis. For a wavelength of $\lambda=0.5 \mathrm{~mm}$ in water at $f=3 \mathrm{MHz}$, this scattering particle appears as point-like scatterer $(d<<\lambda)$.

The scattering object has been mounted on two linear axes which can move the object in the $x-y$ plane. The small scattering object has been mechanically scanned along the $x$-y-plane while measuring the amplitude of the echo signal. Figure 5 shows the sound field based on that approach where the PAUDV was configured for a sound field with a steering angle of $\alpha=7.1$ degree and a focal depth of $F=40 \mathrm{~mm}$, using 64 elements.

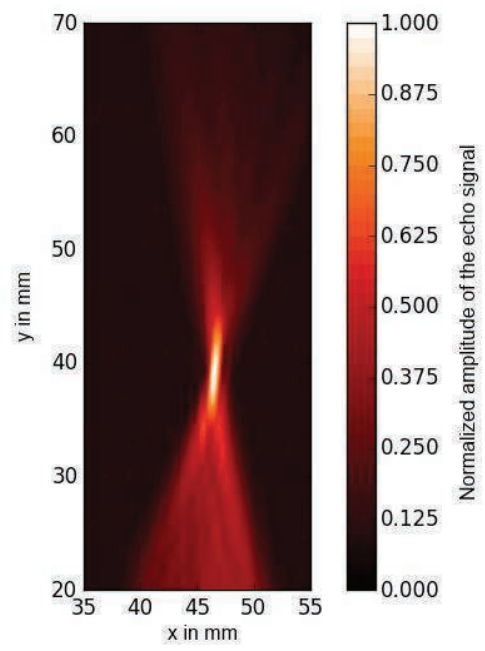

Figure 3: Measured sound field of the phased array sensor with $F=40 \mathrm{~mm}$ and $\alpha=7.1$ degree

The diagram shows a strong directivity of the sound field in very good accordance with the expected behavior. To quantify the achievable resolution of that system configuration, a separate scan along the $x$-axis in a distance of $y=F=40 \mathrm{~mm}$ using a scatter object with a diameter of $d=70 \mu \mathrm{m}$ and no steering of the sound field has been recorded (figure 6). Subsequently, we measured the distance between the two local minima around the amplitude maximum. At $F=40 \mathrm{~mm}$, the PAUDV achieves a resolution of better than $1.5 \mathrm{~mm}$ with the given phased array. The resolution in axial direction (y) is approximately $1.8 \mathrm{~mm}$ for $f=3 \mathrm{MHz}$ with a pulse length of 4 periods and the sound velocity $c_{G a l n S n}$

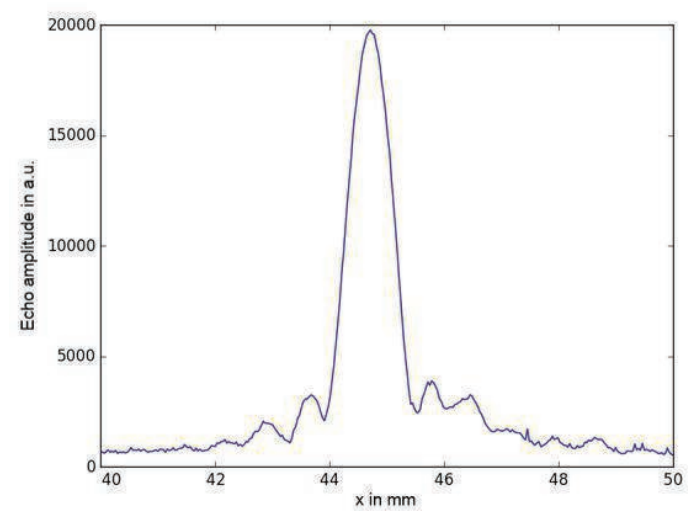

Figure 4: Mechanical scan of the sound field through the focal point along the $x$-axis at a distance of $y=F=40 \mathrm{~mm}$ 


\section{Summary and outlook}

Experimental studies are of great interest in MHD research to complement and refine numerical models and extend the knowledge of the interaction between magnetic fields and conductive fields.

We presented a modular PAUDV system for mapping flows and novel experiments in MHD like two-point correlation measurement of turbulent flows with variable focal points, highresolution scanning and two component measurements through one acoustical access via sound beam steering. The PAUDV achieves a pulse frequency of $60 \mathrm{kHz}$ with an individual programmable beamforming delay resolution of $1.6 \mathrm{~ns}$ for 256 channels. With the designed phased array, the system achieves a resolution of better than $1.5 \mathrm{~mm}$ in lateral direction.

To further improve the performance, it is planned to extend the current TX-beamforming operation with receive beamforming through online signal processing on a FPGA, enabling long-time measurements with both transmit and receive beamforming operation.

A currently planned experiment involves the PAUDV performing flow mapping in a rectangular channel, where the main flow direction is orthogonal to the sensor emission direction. The ultrasound beam is therefore steered to receive Doppler signals containing the main flow velocity component. After a scan with different focus points and the corresponding Doppler velocity estimation, we apply a coordinate transformation algorithm to finally obtain the flow map of the main flow direction. This experiment investigates noninvasive measurements of orthogonally flowing liquids from the view of the sensor, using a clamp-on setup.

\section{Acknowledgement}

The authors like to thank Kevin Mäder for fruitful discussions. The work was partially funded by the DFG (German Research Foundation) with the grant BU 2241/2-1.

\section{References}

[1] Büttner L., Nauber R., Burger M., Räbiger D. Franke S., Eckert S., Czarske J., Meas. Sci. Technol. 24 (2013) 055302, [doi:10.1088/09570233/24/5/055302]

[2] R. Nauber, M. Burger, M. Neumann, L. Büttner, K. Dadzis, K. Niemietz, O. Pätzold, J. Czarske, Exp Fluids (2013) 54:1502 [doi:10.1007/s00348013-1502-x]

[3] V. Schmitz, W.Müller, G.Schäfer, Acoustical Imaging 19 (1992), [doi: 10.1007/978-1-4615-33702_86]
[4] M. Burger, R. Nauber, M. Neumann, L. Büttner, J. Czarske, Technisches Messen: Vol. 79 (2012), No. 9, [doi: 10.1524/teme.2012.0266]

[5] B. E. Treeby, J. Jaros, A. P. Rendell, and B. T. Cox, J. Acoust. Soc. Am. (2012), vol. 131, no. 6 [doi: 10.1121/1.4712021] 